\title{
CONSUMO DE ÁLCOOL EM BINGE E USO DE DROGAS ILÍCITAS ENTRE ADOLESCENTES ESCOLARES
}

\author{
BINGE DRINKING AND ILLICIT DRUG USE AMONG HIGH SCHOOL STUDENTS
}

\section{Jakelline Cipriano dos Santos Raposo ${ }^{\mathrm{a}}$, Ana Carolina Costa ${ }^{\mathrm{b}}$, Paula Valença ${ }^{\mathrm{c}}$, Viviane Colares ${ }^{\mathrm{d}}$, Carolina da Franca ${ }^{\mathrm{e}}$}

\author{
ajakecipriano@gmail.com, bacqueirozcosta@yahoo.com.br, ${ }^{c}$ valensa@gmail.com, ${ }^{d}$ viviane.colares@upe.br, ${ }^{\mathrm{e}} \mathrm{carolina}$. franca@upe.br
} Universidade de Pernambuco - Petrolina (PE), Brasil

Data de recebimento do artigo: 15/08/2015 Data de aceite do artigo: 19/11/2015

\section{RESUMO}

Introduçáo: A prática de beber em binge diminui a capacidade de discernimento e pode aumentar o risco de envolvimento de adolescentes com drogas ilícitas. Objetivos: Investigar a associação entre a prática de beber em binge e o uso de drogas ilícitas (maconha, cocaína e inalantes) em adolescentes escolares. Materiais e métodos: Estudo transversal, com 175 estudantes, de ambos os sexos, matriculados no ensino médio da rede pública de ensino. A seleçáo amostral foi probabilística em duas etapas, com sorteio das escolas e turmas, respectivamente. Os dados foram coletados em abril de $2014 \mathrm{em}$ cinco escolas, utilizando-se a versão validada em português do Youth Risk Behavior Survey. Foram utilizados os módulos sobre consumo de bebidas alcoólicas, uso de maconha e outras drogas. Foram realizadas análises descritivas, com distribuição de frequências e inferencial, através do teste do qui-quadrado para associaçôes. Resultados: A maioria dos adolescentes era do sexo feminino (56,7\%), na faixa etária de 16-19 anos (70,2\%), matriculados na escola regular $(80,7 \%)$ e com renda familiar acima de um salário mínimo $(54,3 \%)$. O consumo de álcool em binge, inalantes e cocaína não apresentou diferença significativa entre os sexos ( $p>0,05)$, ao contrário do uso de maconha, que foi maior nos meninos $(\mathrm{p}<0,05)$. Todos os padróes de uso de drogas foram associados ao consumo de álcool em binge $(\mathrm{p}<0,05)$. Conclusão: A prática de beber em binge entre adolescentes foi associada ao uso de maconha, cocaína e inalantes.

Palavras-chave: Adolescente; comportamento do adolescente; beber em binge; drogas ilícitas.

\section{ABSTRACT}

Introduction: Binge drinking impairs judgment capacity and can lead to illicit drugs use among adolescents. Objectives: Investigate the association between binge drinking and use of illicit drugs (marijuana, cocaine and inhalants) among high school students. Materials and methods: Cross-sectional study with 175 students, from both genders, enrolled in public high school in a two-stage cluster sampling, drawing schools and classes, respectively. Data were collected in April 2014 in five schools, using the validated Portuguese version of the Youth Risk Behavior Survey. Modules were used on alcohol consumption, use of marijuana and other drugs. Descriptive and inferential analyses were carried out with frequencies distribution, using chi-square test. Results: Most of the students were female (56.7\%), aged 16-19 years (70.2\%), regularly enrolled students (80.7\%) and with family income above minimum wage (54.3\%). Binge drinking, inhalants and cocaine use showed no significant difference between the sexes $(p>0.05)$, unlike marijuana use, which was higher on boys $(\mathrm{p}<0.05)$. All drug use patterns were associated with binge drinking. Conclusion: The practice of binge drinking among adolescents was associated with marijuana use, as well as cocaine and inhalants.

Keywords: Adolescent; adolescent behavior; binge drinking; illicit drugs. 


\section{Introdução}

No Brasil, o consumo de álcool é permitido por lei somente aos maiores de 18 anos, entretanto quase $35 \%$ dos adolescentes com idade entre 14 a 17 anos consomem álcool. E desses, 26\% consomem álcool de maneira excessiva, ou seja, em binge $e^{1}$. Essa prática está associada a diversos agravos agudos à saúde, como intoxicaçóes, violência e acidentes ${ }^{2-4} \mathrm{e}$, particularmente nos adolescentes, às alteraçôes neurológicas, pois seu sistema nervoso ainda está em desenvolvimento ${ }^{5-6}$.

O álcool por ser uma droga de fácil acesso ${ }^{7}$, também pode representar uma "porta de entrada" para o uso de maconha e de outras drogas ilícitas ${ }^{8}$. E o uso de mais de uma droga pode potencializar os efeitos negativos no organismo? ${ }^{9}$, que vão desde intoxicações a transtornos de dependência e morte ${ }^{10-13}$.

Diante do exposto, o objetivo do presente estudo foi verificar a associação entre o consumo de álcool em binge e o uso de drogas ilícitas entre estudantes adolescentes da rede pública de ensino.

\section{Casuística e métodos}

Esta pesquisa foi aprovada pelo comitê de ética da Universidade de Pernambuco (CAAE 13800813.7.0000.5207). Os participantes concordaram em participar da pesquisa através da assinatura do termo de assentimento e do termo de consentimento livre e esclarecido por seus pais/responsáveis.

Este foi um estudo transversal e fez parte do projeto maior "Atenção Integral à Saúde dos Adolescentes nos Serviços Públicos de Olinda". Para o cálculo amostral foi utilizado $15 \%$ da amostra total do projeto. A seleção da amostra foi realizada por conglomerado em dois estágios: no primeiro, as escolas foram sorteadas e no segundo, as turmas em cada uma das escolas. Fizeram parte da amostra cinco escolas e 12 turmas do turno diurno.

Os critérios de elegibilidade foram: estudantes de ambos os sexos, na faixa etária de 10 a 19 anos e regularmente matriculados na rede pública estadual de ensino. Foram excluídos os estudantes impossibilitados de responder ao questionário em decorrência de deficiência.

A coleta de dados ocorreu em abril de 2014 por meio da aplicação da versão validada no Brasil do questionário autoadministrado Youth Risk Behavior Survey (YRBS Brasil $)^{14}$, como parte integrante do projeto "Atenção Integral à Saúde dos Adolescentes nos Serviços Públicos de Olinda”. Esse instrumento avalia condutas de risco à saúde de adolescentes e jovens e está dividido em 10 módulos. Para esta pesquisa foram utilizados apenas os módulos de consumo de álcool, uso de maconha e uso de outras drogas (cocaína e inalantes). $\mathrm{O}$ instrumento foi aplicado em sala de aula, sem a presença do professor, por pesquisadores previamente treinados num período de tempo entre 30 e 60 minutos.

Para esta pesquisa foram utilizadas as perguntas sobre a prática de beber em binge e o uso das drogas ilícitas maconha, cocaína e inalantes. As perguntas e suas categorizações estão descritas no Quadro 1.

Os dados foram tabulados com dupla entrada utilizando o software Epidata versáo 3.1 e os erros detectados foram corrigidos. Foi realizada a análise estatística descritiva e inferencial. Para testar as associaçóes foi utilizado o teste do qui-quadrado admitindo-se significância aos valores de $\mathrm{p}<0,05$. As análises foram realizadas usando o SPSS para Windows.

Quadro 1: Descrição das questões utilizadas na pesquisa.

\begin{tabular}{|c|c|c|}
\hline Questóes & $\begin{array}{c}\text { Categorias } \\
\text { originais } \\
\text { Beber álcool em binge }\end{array}$ & $\begin{array}{c}\text { Categorias para } \\
\text { análise }\end{array}$ \\
\hline $\begin{array}{l}\text { "Durante os últimos } \\
30 \text { dias, em quantos } \\
\text { dias você tomou } \\
\text { cinco ou mais doses } \\
\text { de bebida alcoólica } \\
\text { em uma mesma } \\
\text { ocasião?" }\end{array}$ & $\begin{array}{c}\text { Nenhum dia a } 20 \\
\text { dias ou mais }\end{array}$ & $\begin{array}{l}\text { Sim } \\
\text { Não }\end{array}$ \\
\hline \multicolumn{3}{|c|}{ Uso de maconha } \\
\hline $\begin{array}{l}\text { "Durante sua vida, } \\
\text { quantas vezes você } \\
\text { usou maconha?" }\end{array}$ & $\begin{array}{l}\text { Nenhum dia a } 100 \\
\text { ou mais }\end{array}$ & $\begin{array}{c}\text { Nenhum(a) dia/vez } \\
\text { Um ou mais dias/ } \\
\text { vezes }\end{array}$ \\
\hline $\begin{array}{c}\text { "Durante os últimos } \\
30 \text { dias, quantas } \\
\text { vezes você usou } \\
\text { maconha?" }\end{array}$ & $\begin{array}{c}\text { Nenhuma vez a } 40 \\
\text { vezes ou mais }\end{array}$ & $\begin{array}{c}\text { Nenhum(a) dia/vez } \\
\text { Um ou mais dias/ } \\
\text { vezes }\end{array}$ \\
\hline \multicolumn{3}{|c|}{ Uso de cocaína } \\
\hline $\begin{array}{l}\text { "Durante sua vida, } \\
\text { quantas vezes você } \\
\text { usou qualquer } \\
\text { forma de cocaína, } \\
\text { incluindo pó, pedra } \\
\text { ou pasta?" }\end{array}$ & $\begin{array}{c}\text { Nenhuma vez a } 40 \\
\text { vezes ou mais }\end{array}$ & $\begin{array}{c}\text { Nenhum(a) dia/vez } \\
\text { Um ou mais dias/ } \\
\text { vezes }\end{array}$ \\
\hline $\begin{array}{l}\text { "Durante os últimos } \\
30 \text { dias, quantas } \\
\text { vezes você usou } \\
\text { qualquer forma de } \\
\text { cocaína, incluindo } \\
\text { pó, pedra ou pasta?" }\end{array}$ & $\begin{array}{c}\text { Nenhuma vez a } 40 \\
\text { vezes ou mais }\end{array}$ & $\begin{array}{c}\text { Nenhum(a) dia/vez } \\
\text { Um ou mais dias/ } \\
\text { vezes }\end{array}$ \\
\hline \multicolumn{3}{|c|}{ Uso de inalantes } \\
\hline $\begin{array}{l}\text { "Durante sua vida, } \\
\text { quantas vezes você } \\
\text { cheirou cola, respi- } \\
\text { rou conteúdos de } \\
\text { spray aerossol (lança } \\
\text { perfume), ou inalou } \\
\text { tinta ou spray que } \\
\text { deixa 'ligado?" }\end{array}$ & $\begin{array}{c}\text { Nenhuma vez a } 40 \\
\text { vezes ou mais }\end{array}$ & $\begin{array}{c}\text { Nenhum(a) dia/vez } \\
\text { Um ou mais dias/ } \\
\text { vezes }\end{array}$ \\
\hline
\end{tabular}




\section{Resultados}

A amostra foi composta por 175 estudantes, dos quais quatro foram excluídos na fase da análise: três por não informar o sexo e um por náo preencher mais de $20 \%$ do questionário. Dessa forma, 171 questionários foram analisados.

A maioria dos participantes era do sexo feminino (57\%), estava matriculada na escola regular $(80,7 \%)$, informou renda familiar de mais de um salário mínimo $(54,3 \%)$ e encontrava-se na faixa etária de 16 a 19 anos $(70,2 \%)$.

A prática de beber em binge foi informada por 19,3\% dos estudantes e por $65,2 \%$ dos que relataram consumir pelo menos uma dose de álcool nos últimos 30 dias. Entre as drogas ilícitas, o uso na vida de maconha $(10,5 \%)$ superou o uso de inalantes $(6,5 \%)$ e cocaína $(4,1 \%)$. Em relação ao uso atual, a maconha $(4,1 \%)$ também foi mais referida em comparação com a cocaína $(1,2 \%)$. O uso atual de inalantes não foi investigado neste estudo.

$\mathrm{Na}$ análise estratificada por sexo, apenas o uso de maconha na vida apresentou diferença significativa, com uma maior proporção de uso entre os meninos (Tabela 1). Em relação ao consumo do álcool em binge, observou-se associaçáo com o consumo de maconha, cocaína e inalantes (uso na vida e atual) (Tabela 2).

Tabela 1: Distribuição dos participantes de acordo com o sexo, consumo de álcool em binge e uso de drogas ilícitas em Olinda-PE, 2014.

\begin{tabular}{|c|c|c|c|}
\hline \multirow[b]{2}{*}{ Consumo de álcool e drogas } & \multicolumn{2}{|c|}{ Sexo } & \multirow[b]{2}{*}{$\begin{array}{l}\text { Valor } \\
\text { de } p\end{array}$} \\
\hline & $\begin{array}{c}\text { Feminino } \\
\mathrm{N}(\%)\end{array}$ & $\begin{array}{c}\text { Masculino } \\
\text { N (\%) }\end{array}$ & \\
\hline Beber em binge & & & $0,474^{*}$ \\
\hline Nenhum dia & $80(82,5)$ & $57(78,1)$ & \\
\hline Um ou mais dias & $17(17,5)$ & $16(21,9)$ & \\
\hline Uso de maconha na vida & & & $0,009^{\dagger}$ \\
\hline Nenhum dia & $92(94,8)$ & $61(82,4)$ & \\
\hline Um ou mais dias & $05(5,2)$ & $13(17,6)$ & \\
\hline Uso atual de maconha & & & $0,450^{\dagger}$ \\
\hline Nenhuma vez & $94(96,9)$ & $70(94,6)$ & \\
\hline Uma ou mais vezes & $03(3,1)$ & $04(5,4)$ & \\
\hline Uso de cocaína na vida & & & $0,450^{\dagger}$ \\
\hline Nenhuma vez & $94(96,9)$ & $70(94,6)$ & \\
\hline Uma ou mais vezes & $03(3,1)$ & $04(5,4)$ & \\
\hline Uso atual de cocaína & & & $0,847^{\dagger}$ \\
\hline Nenhuma vez & $96(99,0)$ & $73(98,6)$ & \\
\hline Uma ou mais vezes & $01(1,0)$ & $01(1,4)$ & \\
\hline Uso de inalantes na vida & & & $0,421^{\dagger}$ \\
\hline Nenhuma vez & $92(94,8)$ & $67(91,8)$ & \\
\hline Uma ou mais vezes & $05(5,2)$ & $06(8,2)$ & \\
\hline
\end{tabular}

*Qui-quadrado.

†Teste exato de Fischer.
Tabela 2: Associação entre beber em binge e uso de drogas ilícitas em Olinda-PE, 2014.

\begin{tabular}{|c|c|c|c|}
\hline & \multicolumn{2}{|c|}{ Binge drinking } & \multirow{2}{*}{$\begin{array}{l}\text { Valor } \\
\text { de } p\end{array}$} \\
\hline & $\begin{array}{c}\text { Nenhum } \\
\text { dia } \\
\mathrm{N}(\%)\end{array}$ & $\begin{array}{c}1 \text { ou mais } \\
\text { dias } \\
\mathrm{N}(\%)\end{array}$ & \\
\hline Uso de maconha na vida & & & $<0,001^{*}$ \\
\hline Nenhum dia & $130(94,9)$ & $22(66,7)$ & \\
\hline Um ou mais dias & $07(5,1)$ & $11(33,3)$ & \\
\hline Uso atual de maconha & & & $<0,001^{\dagger}$ \\
\hline Nenhuma vez & $137(100,0)$ & $26(78,8)$ & \\
\hline Uma ou mais vezes & $00(0,0)$ & $07(21,2)$ & \\
\hline Uso de cocaína na vida & & & $\mathbf{0 , 0 0 3 ^ { \dagger }}$ \\
\hline Nenhuma vez & $135(98,5)$ & $28(84,2)$ & \\
\hline Uma ou mais vezes & $02(1,5)$ & $05(15,2)$ & \\
\hline Uso atual de cocaína & & & $0,037^{\dagger}$ \\
\hline Nenhuma vez & $137(100,0)$ & $31(93,9)$ & \\
\hline Uma ou mais vezes & $00(0,0)$ & $02(6,1)$ & \\
\hline Uso de inalantes na vida & & & $0,040^{\dagger}$ \\
\hline Nenhuma vez & $130(95,6)$ & $28(84,8)$ & \\
\hline Uma ou mais vezes & $06(4,4)$ & $05(15,2)$ & \\
\hline
\end{tabular}

${ }^{*}$ Qui-quadrado. ${ }^{\dagger}$ Teste exato de Fischer.

\section{Discussão}

A prática de beber em binge foi um comportamento relatado por dois de cada dez adolescentes escolares pesquisados. Essa proporção de adolescentes que relataram beber em binge também foi verificada entre estudantes do ensino médio de 14 a 18 anos de escolas públicas e privadas nas 27 capitais brasileiras, em que $20,9 \%$ relataram consumir álcool em binge nos últimos 30 dias $^{16}$.

$\mathrm{O}$ consumo de álcool em binge foi comum para a maioria dos que relataram consumir pelo menos uma dose de álcool nos últimos 30 dias. Pesquisa realizada com estudantes do ensino médio nos Estados Unidos encontrou resultado similar $(64,2 \%)^{17}$. Esses resultados apontam que a maior parte do consumo de álcool entre adolescentes escolares é feito na forma de binge, e esse padrão de consumo é considerado de risco, pois aumenta rapidamente o nível de álcool no sangue e os efeitos deletérios do álcool de forma aguda.

$\mathrm{O}$ uso de maconha e cocaína (na vida e atual) foi relativamente mais elevado do que o encontrado nos últimos levantamentos nacionais com a população adolescente ${ }^{1,18}$. Sugere-se que essa diferença se deva ao fato desses levantamentos terem sido realizados em escala nacional, o que pode ter distribuído essa prevalência, visto que o consumo de drogas difere por tipo de escola (pública/privada), por cidade e região investigada, com uma 
maior predominância no Sudeste e menor no Norte do País ${ }^{18}$. Em relação ao uso de inalantes, observou-se nesse estudo uma prevalência similar à pesquisa realizada com estudantes de 15 a 19 anos em Minas Gerais, Sudeste do Brasil, no qual 6,4\% dos estudantes da rede pública de ensino relataram o uso de inalantes durante a vida ${ }^{19}$.

A prática de beber em binge, assim como o uso de drogas ilícitas (na vida e atual) não apresentaram diferenças significativas entre os sexos, em concordância com outros estudos realizados com adolescentes ${ }^{19-20}$. Esse ainda é um tema controverso, pois outras pesquisas indicam que o sexo masculino ainda representa um fator de risco para o uso de álcool e drogas lícitas ${ }^{4,21-22}$. Contudo, pesquisa de base populacional alerta para uma tendência do aumento de $87 \%$ em um período de seis anos para o consumo de álcool em binge entre as mulheres do Nordeste Brasileiro, sendo o maior aumento em comparação com outras regiốes brasileiras ${ }^{1}$.

A prática de beber em binge foi associada ao uso de maconha, cocaína e inalantes, concordando com a literatura $^{17,19,23-24}$. Entretanto, outras covariáveis foram analisadas, bem como outros comportamentos de risco ${ }^{17,19,23-24}$, fatores relacionados à escola ${ }^{19,24}$ e à famíliaa ${ }^{19,23}$. O uso de maconha e cocaína na vida não foi investigado nesses estudos e somente um deles avaliou a associaçáo do binge alcoólico com o uso de maconha, inalantes e cocaína ${ }^{17}$.

Esta pesquisa e os estudos citados não verificaram se o uso de drogas ilícitas foi concomitante com o consumo de álcool em binge. Sugere-se que pesquisas futuras investiguem se esse consumo ocorreu de forma simultânea, pois podem existir interaçóes farmacológicas dessas drogas com o álcool - como no caso da cocaína, na qual há formação de um novo metabólito (cocaetileno) com meia-vida superior a da cocaína ${ }^{25}$.

Até o momento, nenhum estudo investigou a associação entre o consumo de álcool em binge e o uso de maconha, cocaína e inalantes entre adolescentes escolares residentes no Nordeste Brasileiro, região de maior consumo de álcool em binge do país ${ }^{1}$. Por isso, o presente estudo considera a investigação dessa regiáo como uma estratégia importante para definição de ações de prevenção e redução de danos na população adolescente. Contudo, entre outras possíveis, consideramos as seguintes limitaçóes desse estudo: a subestimativa da prática de beber em binge para as meninas, como já dito anteriormente; a quantidade de alunos por turma (até 50 alunos) e o tipo de estudo (transversal), que não permite inferir causalidade.

\section{Conclusão}

A prática de beber em binge entre adolescentes foi percentualmente maior entre aqueles que relataram uso de maconha, cocaína (na vida e atual) e inalantes (na vida).

\section{Referências}

1. Laranjeira R, Madruga CS, Pinsky I, Caetano R, Mitsuhiro SS. II Levantamento Nacional de Álcool e Drogas no Brasil (LENAD) - 2012. São Paulo: Instituto Nacional de Ciência e Tecnologia para Políticas Públicas de Álcool e Outras Drogas (INPAD), UNIFESP; 2014 [acesso em 2014 Dez 06]. Disponível em: http://inpad.org.br/wp-content/ uploads/2014/03/Lenad-II-Relat\%C3\%B3rio.pdf

2. Sánchez AS, Martín SR, Vicário MIG, Miranda AV. Episodios de urgencia hospitalaria relacionados com el consumo de alcohol em personas de entre 10 y 30 años de edad en Castilla y León durante el período 2003-2010. Rev Esp Salud Pública. 2012;86(4):409-17.

3. Cisler JM, Begle AM, Amstadter AB, Resnick HS, Danielson $\mathrm{CK}$, Saunders BE. Exposure to interpersonal violence and risk for PTSD, depression, delinquency, and binge drinking among adolescents: data from the NSA-R. J Trauma Stress. 2012;25(1):33-40.

4. Birdsall WC, Reed BG, Huq SS, Wheeler L, Rush S. Alcohol-impaired driving: average quantity consumed and frequency of drinking do matter. Traffic Inj Prev. 2012;13(1):24-30.

5. Lisdahl KM, Thayer R, Squeglia LM, McQueeny TM, Tapert SF. Recent binge drinking predicts smaller cerebellar volumes in adolescents. Psychiatry Res. 2013;211(1):17-23.

6. López-Caneda E, Mota N, Crego A, Velasquez T, Holguín SR, Corral M, et al. Anomalías neurocognitivas asociadas al consumo intensivo de alcohol (binge drinking) en jóvenes y adolescentes: una revisión. Adicciones. 2014;26(4):334-59.

7. Romano M, Duailibi S, Pinsky I, Laranjeira R. Pesquisa de compra de bebidas alcoólicas por adolescentes em duas cidades do estado de São Paulo. Rev Saúde Pública. 2007;41(4):495-501.

8. Degenhardt L, Dierker L, Chiu WT, Medina-Mora ME, Neumark Y, Sampson N, et al. Evaluating the drug use "gateway" theory using cross-national data: consistency and associations of the order of initiation of drug use among participants in the WHO World Mental Health Surveys. Drug Alcohol Depend. 2010;108(1-2):84-97.

9. Bava S, Frank LR, McQueeny T, Schweinsburg BC, Schweinsburg AD, Tapert SF. Altered white matter microstructure in adolescent substance users. Psychiatry Res. 2009;173(3):228-37.

10. Degenhardt L, Ferrari AJ, Calabria B, Hall WD, Norman RE, McGrath J, et al. The global epidemiology and contribution of cannabis use and dependence to the global burden of disease: results from the GBD 2010 study. PLos ONE. 2013;8(10):e76635. 
11. Haberstick BC, Young SE, Zeiger JS, Lessem JM, Hewitt JK, Hopfer CJ. Prevalence and correlates of alcohol and cannabis use disorders in the United States: results from the national longitudinal study of adolescent health. Drug Alcohol Depend. 2014;136:158-61.

12. Santos S, Brugal MT, Barrio G, Castellano Y, DomingoSalvany A, Espelt A, et al. Assessing the effect of patterns of cocaine and alcohol use on the risk of adverse acute cocaine intoxication. Drug Alcohol Rev. 2012;31(4):439-46.

13. Degenhardt L, Hall W. Extent of illicit drug use and dependence, and their contribution to the global burden of disease. Lancet. 2012;379(9810):55-70.

14. Guedes DP, Lopes CC. Validação da versão brasileira do Youth Risk Behavior Survey 2007. Rev Saúde Pública. 2010;44(5):840-50.

15. Kann L, Kinchen S, Shanklin SL, Flint KH, Hawkins J, Harris WA. Youth Risk Behavior Surveillance - United States, 2013. MMWR CDC surveill summ [periódico na internet]. 2014 Jun [acesso 2014 Set 10];63(SS04):1-168. Disponível em: http://www.cdc.gov/mmwr/pdf/ss/ss6304.pdf.

16. Sanchez ZM, Locatelli DP, Noto AR, Martins SS. Binge drinking among Brazilian students: a gradient of association with socioeconomic status in five geo-economic regions. Drug Alcohol Depend. 2013;127(1-3):87-93.

17. Miller JW, Naimi TS, Brewer RD, Jones SE. Binge drinking and associated health risk behaviors among high school students. Pediatrics. 2007;119(1):76-85.

18. Carlini ELA, editor, Noto AR, Sanchez ZVDM, Carlini CMA, Locatelli DP, Abeid LR, et al. VI Levantamento sobre o consumo de drogas psicotrópicas entre estudantes do ensino das redes pública e privada de ensino nas $27 \mathrm{ca}-$ pitais brasileiras - 2010. São Paulo: Centro Brasileiro de Informaçôes sobre Drogas Psicotrópicas (CEBRID); 2010 [acesso em 2015 Fev 18]. Disponível em: http://www.cebrid.epm.br/index.php.

19. Silva-Oliveira F, Jorge KO, Ferreira EF, Vale MP, Kawachi I, Zarzar PM. The prevalence of inhalant use and associated factors among adolescents in Belo Horizonte, Brazil. Cien Saude Colet. 2014;19(3):881-90.

20. Meneses C, Markez I, Romo N, Uroz J, Rua A, Laespada T. Diferencias de género en el consumo diario de tabaco e intensivo de alcohol en adolescentes latinoamericanos en tres areas españolas (Andalucia, Madrid y País Vasco). Rev Asoc Esp Neuropsiquiatr. 2013;33(119):525-35.

21. Locatelli D, Sanchez Z, Opaleye E, Carlini C, Noto A. Socieconomic influences on alcohol use patterns among private school students in São Paulo. Rev Bras Psiquiatr. 2012;34(2):193-200.

22. Harakeh Z, de Looze ME, Schrijvers CTM, van Dorsselaer SA, Vollebergh WA. Individual and environmental predictors of health risk behaviours among Dutch adolescents: the HBSC study. Public Health. 2012;126(7):566-73.

23. Stickley A, Koyanagi A, Koposov R, McKee M, Roberts B, Murphy A et al. Binge drinking among adolescents in Russia: prevalence, risk and protective factors. Addict Behav. 2013;38(4):1988-95.

24. Patrick ME, Schulenberg JE, Martz ME, Maggs JL, O'Malley PM, Johnston LD. Extreme binge drinking among 12th-grade students in the United States: prevalence and predictors. JAMA Pediatr. 2013;167(11):1019-25.

25. Harris DS, Everhart ET, Mendelson J, Jones RT. The pharmacology of cocaethylene in humans following cocaine and ethanol administration. Drug Alcohol Depend. 2003;72(2):169-82.

\section{Como citar este artigo:}

Raposo JCS, Costa AC, Valença P, Colares V, Franca C. Consumo de álcool em binge e uso de drogas ilícitas entre adolescentes escolares. Rev. Aten. Saúde. 2016;14(48):27-31. 